\title{
Écriture scientifique et évolution des croyances, des connaissances ou des conceptions des enseignants en reprise d'étude
}

\author{
Catherine Delarue-Breton \\ Université Paris Est, F-94010 Créteil Cedex \\ Laboratoire CIRCEFT-Escol, EA 4384, Universités Paris 8 et Paris Est Créteil \\ catherine.delarue-breton@u-pec.fr
}

\begin{abstract}
Résumé. Si l'on s'accorde assez unanimement - c'est à dire aux plans simultanément scientifique et institutionnel - à reconnaitre que l'initiation à la recherche est devenue nécessaire dans la formation des enseignants, les modalités de cette initiation, ses visées et ses effets font cependant peu l'objet, encore, d'un consensus, et dans certains cas, divisent. Nous avons pu étudier à diverses reprises, à partir de différentes versions des mémoires, le rôle de l'écriture scientifique dans l'appropriation des concepts théoriques par les étudiants de master MEEF (Métiers de l'Enseignement, de l'Éducation, de la Formation) en formation initiale, autrement dit par les étudiants issus de licence, se destinant au métier de professeur des écoles. Nous avons ainsi pu mettre au jour d'une part la diversité de ces modes d'appropriation conceptuelle, et, en corrélation avec celle-ci, la diversité des modes d'analyse des situations scolaires, et d'autre part des formes d'évolution ou de stagnation des croyances, connaissances, conceptions de ces futurs enseignants sur le métier, sur les élèves, sur les disciplines, sur l'apprentissage. Notre propos est ici de nous poser des questions comparables, concernant le rapport entre appropriation théorique et évolution des conceptions non pas d'enseignants en formation initiale, mais d'enseignants titulaires en reprise d'étude, parfois eux-mêmes formateurs, inscrits en master MEEF avec validation des acquis. Nous questionnons ainsi le rôle de la recherche et de l'écriture scientifique dans la formation continue des enseignants.
\end{abstract}

\begin{abstract}
If the scientists and the school institution agree to say that the research has become necessary in the training of the teachers, its methods and its effects are still underestimated. We may notice, from the study of various versions of these master's theses, how the scientific writing enables the students who plan to work in teaching professions to make these theoretical concepts theirs, during the initial training. We were able to show that these appropriations are differently made, but also that teachers' believes or conceptions about the job, the pupils, the school subjects can evolve or stagnate. We ask comparable issues about the theoretical appropriation by teachers, but in further teacher training. So, we question the role of the research and the scientific writing for further teacher training.
\end{abstract}




\begin{abstract}
«Où maintenant? Quand maintenant? Qui maintenant? Sans me le demander. Dire je. Sans le penser. Appeler ça des questions, des hypothèses. Aller de l'avant [...] Je ne serai pas seul les premiers temps. [...] Je vais avoir de la compagnie. Pour commencer. Quelques pantins. Je les supprimerai par la suite. Si je peux.
\end{abstract}

Samuel Beckett (2004/1953), L'innommable. Paris : Minuit, 7-8.

Si l'on s'accorde assez unanimement - c'est à dire aux plans simultanément scientifique et institutionnel - à reconnaitre que l'initiation à la recherche est devenue nécessaire dans la formation des enseignants, les modalités de cette initiation, ses visées et ses effets font cependant peu l'objet, encore, d'un consensus, et dans certains cas, divisent. Nous avons pu étudier à diverses reprises, à partir de différentes versions des mémoires, le rôle de l'écriture scientifique dans l'appropriation des concepts théoriques par les étudiants de master $\mathrm{MEEF}^{1}$ en formation initiale (voir notamment Delarue-Breton, 1014 ; DelarueBreton \& Crinon, 2015 ; Delarue-Breton, 2016 ; Delarue-Breton \& Dolignier, 2016). Nous avons ainsi pu mettre au jour d'une part la diversité de ces modes d'appropriation, et, en corrélation avec celle-ci, la diversité des modes d'analyse des situations scolaires, et d'autre part des formes d'évolution ou de stagnation des croyances, connaissances, conceptions de ces futurs enseignants sur le métier, sur les élèves, sur les disciplines, sur l'apprentissage.

Notre propos est ici de nous poser des questions comparables, concernant le rapport entre appropriation théorique et évolution des conceptions, non plus à propos d'enseignants en formation initiale, mais à propos d'enseignants titulaires en reprise d'étude, parfois eux-mêmes formateurs, inscrits en master MEEF avec validation des acquis. Nous questionnons ainsi le rôle de la recherche et de l'écriture scientifique dans la formation continue des enseignants.

\title{
1 Contexte de la recherche : soubassements théoriques et corpus
}

Élaborer une pensée propre à partir des apports théoriques d'autrui, processus qui relève de l'au(c)torisation du sujet écrivant (Delarue-Breton, 2014a), suppose un double mouvement d'intégration dans une communauté discursive et de distanciation vis-à-vis de cette communauté. L'écriture scientifique est au fondement de ce processus, au cours duquel l'auteur de mémoire s'approprie des savoirs déjà disponibles sur son thème ou sa question de recherche, et les redéploie à des fins d'analyse. Les appuis théoriques que nous convoquons pour cette contribution concernent donc d'abord les propriétés de l'écriture scientifique.

Notre visée est cependant d'évaluer l'impact de ce double mouvement de la pensée scientifique sur l'évolution des croyances, connaissances ou conceptions que les auteurs de mémoire ont des différents aspects du métier. Nous nous appuierons donc pour penser cette question complexe sur une seconde série d'apports théoriques concernant précisément ces croyances et autres conceptions. 


\subsection{Les propriétés de l'écriture scientifique}

Celles-ci ont été étudiées à partir de diverses approches, analysant aussi bien les textes scientifiques de chercheurs confirmés que ceux de débutants. Ce sont ces derniers travaux que nous retenons pour cette étude, et plus particulièrement ceux qui concernent les propriétés des textes produits par des chercheurs en formation, tenus d'écrire un mémoire (de doctorat ou de master).

\subsubsection{Approche énonciative des écrits des étudiants}

F. Boch notamment, dans un article consacré à l'écriture scientifique des doctorants, et qui étudie des caractéristiques énonciatives du genre écrit scientifique (Boch, 2013), met en avant trois domaines principaux susceptibles montrer des variations dans les positionnements d'auteur. Elle qualifie ces domaines de « zones clignotantes de l'écrit scientifique, en ce qu'elles tiennent une place importante dans les études descriptives des "manières de faire" des chercheurs [...] et sont également reconnues comme sources de difficultés chez les étudiants [...]. » (ibid., 545). Ces dimensions sont les suivantes :

- Positionnement de l'auteur dans un texte à visée objectivante

- Légitimation de l'objet d'étude

- Intégration des sources

Pour le premier item, il s'agit de mieux comprendre comment l'auteur de mémoire s'implique personnellement et prend position dans une recherche à visée objective et généralisante, position parfois perçue à tort par les étudiants comme relevant de la neutralité. Dans une perspective du même ordre, nous avons nous-même montré (Delarue-Breton, 2016) dans quelle mesure les étudiants sont confrontés dans ce travail d'écriture spécifique à la nécessité de créer eux-mêmes, à des fins de renouvèlement scientifique, ce qui l'a déjà été par d'autres, ailleurs et auparavant - nous reprenons à Winnicott (1975/1971) la formule créer le déjà-là - autrement dit de s'approprier des savoirs théoriques en vue notamment de les exploiter dans l'analyse des données, et non de les mentionner à des fins de simple restitution. Cet usage propre des savoirs théoriques est une caractéristique souvent implicite de l'accès au niveau Master ${ }^{2}$ du cursus universitaire : alors que l'étudiant de licence voit ses écrits valorisés le plus souvent sur le critère principal de la restitution convenable (formulation adaptée, absence de contresens, connaissance de l'environnement du concept etc.), il est attendu de l'étudiant de master qu'il exploite luimême - donc qu'il intériorise - ces savoirs pour nourrir et mener à terme son propre questionnement, ce qui entraine un changement de posture souvent peu enseigné à ce niveau.

Le deuxième item concerne la justification de l'objet de recherche, qui amène cette fois-ci, dans un mouvement inverse à celui précédemment évoqué, l'étudiant à donner une légitimation externe à son propre choix, en s'appuyant par exemple sur d'autres travaux en cours sur le même thème, ou, à l'opposé, sur le caractère manquant de tels travaux dans la production scientifique, ou encore sur les contradictions présentes dans les publications scientifiques, qui supposent de continuer à creuser la question, à légitimer telle ou telle approche etc.

Le troisième item, concernant l'intégration des sources, se décline en trois possibilités (Boch, op. cit., 558) :

- Citation directe avec guillemets

- Reformulation

- Évocation

Dans le premier cas (mode de référencement privilégié des chercheurs débutants), la citation reprend l'énoncé évoqué sans modification, limitant ainsi ce que Frier (1998) nomme les risques interprétatifs. Dans le deuxième cas, la reformulation du propos d'autrui permet la prise en compte de la parole de l'auteur du mémoire : elle est donc susceptible de traduire davantage le positionnement de l'auteur du mémoire ou son implication vis-à-vis du savoir mentionné, mais comporte de ce fait un risque d'interprétation plus grand. Dans le troisième cas, l'auteur du mémoire évoque le savoir concerné sans le définir ou en résumer la teneur; il peut aussi (Delarue-Breton, 2016) faire allusion - sans mentionner à nouveau le nom du ou des auteurs source(s) - à un concept suffisamment cité dans son texte (ou plus 
largement suffisamment partagé au sein de la communauté discursive) pour que sa référence ne fasse pas problème.

Nous verrons plus loin que l'étude de ces items dans notre corpus permet de mieux comprendre la manière dont les auteurs de mémoire s'impliquent dans la réflexion scientifique, et s'y déplacent ; ceux-ci ne sont cependant pas suffisants pour rendre compte des tenants et aboutissants de l'appropriation conceptuelle au sein du genre spécifique que représente le mémoire de master MEEF.

\subsubsection{Le genre mémoire de master MEEF}

Le mémoire de master MEEF, partie prenante de la formation des enseignants depuis la publication de l'arrêté du 27 août $2013^{3}$, s'inscrit dans un paysage universitaire complexe, où le type d'écrit mémoire renvoie à des formes et des exigences très hétérogènes, et à des traditions opposées.

En termes de tradition, nous pouvons évoquer la séparation, à l'université, entre «mémoire de recherche» et «mémoire professionnel», le premier invitant son auteur à une poursuite d'études et ouvrant éventuellement la voie à une carrière universitaire, le second signant au contraire la fin des études et ouvrant à une carrière professionnelle en dehors de l'université. Or, l'article 19 de l'arrêté du 27 août 2013 précise notamment que « chaque étudiant réalise un mémoire de master qui doit avoir un contenu disciplinaire et de recherche en relation avec la finalité pédagogique et les pratiques professionnelles ». Le cadre institutionnel du mémoire de master MEEF le définit donc bien comme un mémoire relevant simultanément du scientifique et du professionnel, ce qui vient revisiter les conceptions qui prévalaient jusqu'alors à l'université.

Du côté de la formation des enseignants, assurée depuis 1990 et jusqu'en 2013 par les IUFM ${ }^{4}$, il s'agissait également pour les futurs enseignants ou enseignants en formation continue de produire un mémoire dit «professionnel », dont le genre s'est avéré très hétérogène, aussi bien entre les académies qu'au sein d'une même académie (voir notamment Crinon, 2003; Crinon \& Guigue, 2004 ; Crinon \& Guigue, 2006). Ce sont donc trois types de mémoires antérieurs au mémoire de master actuel qui contribuent à brouiller le genre discursif actuel du mémoire de master MEEF.

Pour leurs auteurs, il s'agit selon nous (voir notamment Delarue-Breton, 2014b) de produire un mémoire qui s'élabore conjointement à une recherche réelle, nous entendons par là un mémoire qui vise la compréhension de phénomènes, et qui réponde aux exigences scientifiques du mémoire de master, au plan théorique comme au plan méthodologique. Mais ce mémoire doit également porter sur un objet explicitement professionnel, que celui-ci concerne des objets «micro » (par exemple l'action de l'enseignant dans sa classe, les apprentissages des élèves, les spécificités disciplinaires etc.), des objets «méso » (par exemple la politique des circonscriptions, les prescriptions institutionnelles, les pratiques d'établissement etc.) ou des objets «macro» (par exemple les politiques éducatives nationales ou internationales, la coéducation etc.). Les possibilités en la matière sont donc fort nombreuses, et toutes les disciplines scientifiques qui s'intéressent à l'éducation, l'enseignement ou la formation sont ici potentiellement concernées, et aucune n'est privilégiée ou n'a vocation à l'être plus qu'une autre (ibid., 51).

Notons enfin que le public concerné, constitué d'enseignants en formation initiale, mais aussi d'enseignants ou de formateurs d'enseignants en formation continue ou en reprise d'étude, s'initie lors de l'élaboration du mémoire à un nouveau mode de socialisation universitaire, susceptible de lui permettre de construire ou de renouveler son identité professionnelle, en s'appropriant de nouveaux modes scientifiques - d'analyse des situations en lien avec le métier.

L'écriture d'un mémoire de master MEEF entraine donc potentiellement des tensions entre trois postures, susceptibles, si elles sont reconnues ou identifiées, de dynamiser la réflexion entreprise, ou de la compromettre, si ces postures se succèdent ou alternent de manière aléatoire, sans s'orchestrer (DelarueBreton, 2014b) :

- La posture d'étudiant (restitution de savoir) 
- La posture d'apprenti-chercheur (production de savoir)

- La posture d'enseignant (transmission de savoir)

Ces différentes formes de rapport au(x) savoir(s), parce qu'elles convoquent aussi des savoirs produits et formulés ailleurs, nous conduisent à prendre en compte les différentes formes de dialogisme susceptibles d'être attestées dans les mémoires.

\subsubsection{Formes du dialogisme : entre hésitation et négociation dialogique}

$\mathrm{Au}$ delà des formes de dialogisme présentes dans tout acte de parole, la nécessité, pour un auteur de mémoire, de dialoguer avec les auteurs qui l'ont précédé pour élaborer son cadre théorique, et s'approprier les savoirs déjà disponibles sur sa question de recherche, entraine en effet des formes spécifiques de dialogisme qu'il convient de préciser. Nous entendons par dialogisme le feuilletage des voix qui traversent le propos du mémoire, que ces voix soient maitrisées (on parlera plutôt dans ce cas de polyphonie), ou qu'elles ne le soient pas (voir notamment sur ces distinction Bres, 2005).

Ce dialogisme, qui convoque explicitement ou plus indirectement des positions théoriques lui préexistant, se double par ailleurs, puisque nous parlons de mémoires de master, d'une préoccupation ou d'un dialogue plus souterrain avec l'instance évaluative que peut représenter le jury, ou, plus largement, le lecteur. On peut ainsi considérer que certains glissements énonciatifs attestent de ce dialogue-là, et traduisent les représentations qui peuvent être celles de l'auteur du mémoire concernant le genre d'écrit attendu, auquel il tente, dans une certaine mesure, de se conformer (Delarue-Breton, 2014a). De ce point de vue, la valeur accordée aux savoirs théoriques convoqués, et leur rôle dans la recherche conduite et, conjointement, dans l'élaboration du mémoire, nous apparaissent fondamentaux, et donc méritent d'être étudiés au même titre que les trois «zones clignotantes» que définit F. Boch, à savoir le positionnement énonciatif, l'enjeu de la recherche et l'intégration des sources.

C. Donahue, pour caractériser la manière dont l'étudiant écrivant - qu'elle nomme sujet textuel et qu'elle définit comme pluri-appartenant (Donahue, 2001) - travaille les tensions auxquelles il est assujetti, parle de négociation dialogique, négociation qu'elle définit comme une lutte féconde au sein d'un espace discursif (Donahue, 2004). Tout en nous inscrivant dans cette conception, nous considérons que si pour certains auteurs de mémoires on peut en effet parler de négociation dialogique - féconde, donc - il apparait que pour d'autres, il semble plus approprié de parler d'hésitation dialogique, afin de mettre en évidence le ballotage entre les positions discursives dont les auteurs font l'objet, et non l'orchestration maitrisée des discours.

Enfin, au delà du dialogue avec les sources théoriques et avec le jury, ou plus largement le lecteur, on observe également dans ces mémoires un dialogue de l'auteur du mémoire avec lui-même, qui nous parait particulièrement remarquable. Rabatel (2013), pour caractériser le dialogue d'un énonciateur avec un point de vue externe, parle d'hétérodialogisme ; on pourrait donc parler d'hétérodialogisme pour décrire le dialogue d'un auteur de mémoire avec un auteur source. Pour caractériser le dialogue d'un énonciateur avec lui-même, il parle d'autodialogisme : l'autodialogisme se caractérise donc par un redoublement du dire par le même énonciateur, traduisant un changement de point de vue, ou la confirmation d'un point de vue antérieurement exprimé.

Les deux formes, hétéro et autodialogisme, sont attestées dans les mémoires, et parfois même au sein du même propos, qui peut faire l'objet d'un feuilletage interprétatif, susceptible de rendre compte du feuilletage discursif qui le constitue. Cet autodialogisme nous parait toutefois particulièrement digne d'intérêt, dans la mesure où il est susceptible de rendre compte aussi de l'évolution des conceptions, connaissances et croyances des enseignants, que nous cherchons également à décrire.

\subsection{Conceptions, connaissances, croyances des enseignants}

Nombreuses sont les recherches concernant les conceptions, connaissances, croyances, représentations, doxas etc., des enseignants, et reprises dans la note de synthèse rédigée par Crahay et al. (2010). Si ces 
travaux divergent sur un certain nombre de points, concernant par exemple le rapport possible entre croyances et pratiques, un consensus relatif semble être établi sur le fait qu'il est peu aisé de définir le terme de croyance (belief, dans les travaux anglophones, qui représentent la majorité d'entre eux), et qu'il est peu aisé de distinguer ce qui est croyance de ce qui est connaissance (knowledge). D'autre part, les auteurs indiquent qu'il est difficile - mais pas impossible - de faire évoluer ces croyances, qui sont multidimensionnelles et multifonctionnelles, et que le développement professionnel des enseignants, indissociable de leur développement personnel, laisse paraitre un mélange de stabilité et de variabilité (ibid., 95).

Crahay et al. précisent par ailleurs que si la littérature concernant ce thème abonde en termes différents, ces termes sont pour autant assez peu différenciables (ibid. 86), voire interchangeables. En ce qui nous concerne, nous retiendrons désormais le terme unique de conception(s), qui nous parait plus pertinent que les autres dans le contexte qui est le nôtre, pour deux raisons.

D'une part, parce qu'il permet de rendre compte du caractère simultanément singulier et partagé, construit - voire argumenté - et spontané de ces points de vues, ce que ne permettent ni le terme croyances, qui renvoie essentiellement à leur dimension idéologique, conjecturale ou peu fondée, ni le terme connaissances, qui renvoie inversement trop exclusivement à leur dimension positive, factuelle, ou empirique.

D'autre part et surtout, nous tentons de mettre en relation les effets des usages de concepts théoriques par les auteurs de mémoires sur leurs mouvements de pensée concernant les objets professionnels qui sont les leurs. Il nous parait ainsi légitime d'employer deux termes de même étymologie, pour restaurer cette continuité qui apparait dans les mémoires entre représentations antérieures et concepts convoqués, autrement dit entre concepts et conceptions. Il apparait en effet que dans certains cas, l'appropriation des concepts permet le renouvèlement des conceptions.

\subsection{Le corpus}

Le corpus sur lequel se fonde la recherche que nous menons depuis trois ans sur le rôle de l'écriture scientifique dans l'évolution du rapport au(x) savoir(s) des enseignants ou futurs enseignants est un corpus empirique constitué de mémoires de $\mathrm{M} 2^{5}$, de notes de recherches de M1 et d'écrits intermédiaires de mémoires ou notes de recherche. Les auteurs de ces mémoires appartiennent à deux types de population : d'une part, une population en formation initiale constituée d'étudiants titulaires d'une licence ou d'un diplôme considéré équivalent, qui préparent simultanément le concours de professeur des écoles et le master, et qui se forment au métier d'enseignant ; d'autre part, une population en formation continue ou en reprise d'étude, constituée d'enseignants du premier degré ou de formateurs du premier degré titulaires et en poste, désireux d'obtenir le diplôme de master pour diverses raisons : obtention d'un niveau de diplôme au moins égal aux enseignants nouvellement recrutés ou qu'ils ont à former ; évolution de carrière ; entrée dans la recherche.

Le corpus sur lequel s'appuie la présente contribution est constitué plus particulièrement de quatre mémoires terminaux et soutenus avec succès, produits par des formateurs/trices du premier degré, qui sont conseillers/ères pédagogiques de circonscription (désormais CPC), directeur/trice d'école d'application (désormais DEA) ou inspecteurs/trices de l'Éducation nationale (désormais IEN).

Ce mémoire, qui a pour objet de présenter dans l'ensemble de ses dimensions la recherche menée par l'étudiant, est donc un document dont le volume attendu est de 100.000 signes environ. Les thématiques de recherche des mémoires retenus concernent le développement professionnel des enseignants (pour le mémoire que nous appellerons document 1), la perception des enjeux d'apprentissage par les élèves au sein des interactions langagières scolaires (pour le mémoire que nous appellerons document 2), le rôle de la reformulation enseignante dans la perception des enjeux d'apprentissage par les élèves (pour le mémoire que nous appellerons document 3) et la prise en charge des enjeux d'apprentissage dans le discours pédagogique en maternelle (pour le mémoire que nous appellerons document 4). 
Nous avons retenu ces quatre documents pour plusieurs raisons. La première tient à l'intérêt que nous portons actuellement à l'introduction de la formation à la recherche et à l'écriture scientifique des enseignants titulaires, qui fait encore peu l'objet d'investigations. La population ainsi définie est une population de volontaires, qui, s'ils découvrent seulement au cours de la formation la nature spécifique de la recherche qu'ils devront eux-mêmes entreprendre, et parfois avec une certaine surprise pour une partie non négligeable d'entre eux, se sont cependant engagés de leur propre chef dans cette démarche. La deuxième raison est qu'il s'agit de quatre formateurs, qui attendent de cette expérience de recherche, pour autant que nous puissions en juger, des évolutions ou transformations dans leur rapport à un métier qu'ils maitrisent, et pour lequel ils ont été reconnus comme particulièrement compétents, puisqu'ils sont tous titulaires du Certificat d'Aptitude aux Fonctions d'Instituteur/Professeur des Écoles Maitre Formateurs (CAFIPEMF). La troisième est qu'ils représentent des formateurs de statuts différents : CPC, DEA, IEN.

L'analyse de ces quatre documents a été conduite à partir de critères centrés d'une part sur les lieux du mémoire susceptibles de voir apparaitre des changements de conception, d'autre part sur les modalités d'écriture du texte et plus particulièrement les modalités énonciatives attestant de formes variées de prise en charge de ces changements de conception, enfin sur l'usage que font les auteurs des concepts convoqués (Delarue-Breton \& Crinon, 2015).

Précisons enfin que nous avons étudié ces travaux d'auteurs relevant de la formation continue, afin de vérifier que le point de vue selon lequel les conceptions, connaissances et croyances des enseignants sont susceptibles d'évoluer d'autant plus en profondeur que les enseignants ont pu les confronter à des savoirs théoriques en formation initiale (Crahay et al., 2010), est également vrai pour la formation continue. Le travail que nous avons conduit nous amène à considérer que la confrontation entre savoirs théoriques et conceptions (d'autant plus fortes qu'elles sont ancrées dans une expérience professionnelle d'enseignement - et de formation - déjà conséquente) peut, à certaines conditions, produire des renouvèlements importants aussi en formation continue, nous y reviendrons.

\section{2 Écriture et évolution des conceptions : quelques résultats}

L'analyse de ces différents écrits a permis de mettre en évidence des mouvements ou déplacements de conception qui se traduisent dans les mémoires de manière aussi bien consciente qu'inconsciente, ou moins consciente. Ces mouvements apparaissent dans différents lieux du mémoire, et se manifestent selon des modalités scripturales et conceptuelles variables. Ils peuvent être plus ou moins affirmés, plus ou moins discrets.

\subsection{Des conceptions initiales évoquées dans les mémoires}

On observe tout d'abord que des conceptions antérieures, qui prévalaient en amont de l'élaboration du mémoire, sont souvent mentionnées, parfois dès les premières pages, en guise d'introduction de la question de recherche, parfois dans d'autres lieux du mémoire. Cette présence de conceptions initiales dit quelque chose du positionnement de l'auteur et de son implication personnelle dans la recherche, mais parle également de la manière dont se légitime l'objet de la recherche.

Précisions que nous ne prenons pas ici en compte le caractère potentiellement construit ou revisité de ces conceptions initiales : il se peut que leur mise en texte ait entrainé une relecture ou reconfiguration de celles-ci, et que leur mention dans le texte les ait formalisées au delà de la manière dont elles pouvaient l'être auparavant. Mais nous pensons néanmoins que ce n'est pas toujours le cas (nous le montrerons), et que quand cela l'est, leur formalisation dans le mémoire nous donne accès à ce que leurs auteurs considèrent comme étant des déplacements dans leurs conceptions, et que de ce fait, ces considérations sont en elles-mêmes dignes d'intérêt. 


\subsubsection{Une présence récurrente}

On observe ainsi dans l'introduction de chacun de ces quatre documents des propos qui parlent d'un « avant » la recherche, formulés de manière explicite :

« Notre expérience, en tant qu'enseignante, et des arguments théoriques, ont forgé une conviction. [...] Un a priori a animé notre recherche : l'explicitation et donc la réflexivité facilite voire génère l'apprentissage.

Nous pensions que l'enseignant, comme l'élève, vit son métier, puis il le perçoit avec de plus en plus de distance et d'objectivité pour enfin, en mettant en mots sa pratique, parvenir à la penser, la concevoir. » [Doc. 1 p.6, souligné par nous]

«Les élèves ont réellement besoin de l'explicitation par le maitre de l'implicite. Ils différencieront ainsi le "faire" de "l'apprendre". Si cette recommandation inscrite en synthèse de nombre de mes rapports me semble fondée pour la plupart des élèves, à ce stade de ma réflexion il me semble que cela n'est pas toujours aussi simple. » [Doc.2 p.6, souligné par nous]

«Depuis que je suis enseignante, j'ai toujours été convaincue de l'importance du discours dans la mise en place d'une ambiance de classe favorable aux apprentissages des élèves ainsi que dans la mise en œuvre de situations d'apprentissages efficaces. » [Doc. 3 p.4, souligné par nous]

«La question de l'inégalité scolaire a toujours été une préoccupation majeure au travers des différents postes que j'ai occupés. Je pense que l'école maternelle peut jouer un rôle primordial dans la lutte contre l'échec scolaire et les inégalités. » [Doc. 4 p.4, souligné par nous]

Dans les quatre cas, les déplacements dont ces conceptions feront l'objet relèvent de l'affinement, de la nuance, de la complexification et non du rejet ou de la remise en cause radicale. Ce point nous semble important, car il permet une évolution de l'identité professionnelle sans doute plus plus harmonieuse ou aisée que si elle était fondée sur le destruction ou l'éradication des conceptions antérieures : la rupture n'est potentiellement envisageable que si elle s'inscrit aussi dans une certaine continuité, qui ne rend pas vaine l'expérience.

Ainsi, dans ces quatre cas, ces conceptions se réfèrent à la pratique professionnelle dans laquelle elles disent s'enraciner ; mais elles sont aussi présentées comme au fondement de la recherche menée au sein du mémoire, contribuant ainsi largement à la légitimation de l'objet de recherche. L'auteur du document 1 donne des précisions sur l'élaboration de son questionnement :

\footnotetext{
«Alors que notre phase de recherche exploratoire nous menait vers la confirmation de cette conjecture, nous avons pourtant découvert que l'étude des conditions favorables à l'activité réflexive n'était pas suffisante.

Le travail de recherche scientifique débutait avec la prise de conscience que le réel de l'activité ne surgit pas spontanément dans le discours des enseignants, même si les conditions favorables sont réunies. Des obstacles gênent la lecture que l'enseignant a du réel, son regard est orienté. » [Doc. 1 p.7]
}

Le document 3 rend explicite la nécessité de la recherche, et met ainsi en évidence ce qui conduit à passer de l'expérience professionnelle à la recherche :

« Ma fonction m'a rarement permis d'observer des maitres chevronnés en situation. Cette recherche m'a donc permis de comparer les types et les fréquences d'utilisation 
des reformulations des enjeux d'apprentissage dans les discours des enseignants novices et des enseignants experts. » [Doc. 3 p. 4]

De même, le document 4 mentionne explicitement l'origine professionnelle du questionnement :

\begin{abstract}
«Les pratiques enseignantes que j'ai observées en tant que conseillère pédagogique lors de mes visites de classe m'ont conduite à m'interroger sur le rôle du langage magistral dans le développement des compétences langagières des élèves de maternelle, notamment lors d'un travail en atelier. » [Doc. 4 p.6]
\end{abstract}

Quant au document 2, il atteste pour sa part d'une conception antérieure effectivement formalisée bien en amont de la recherche, et non à l'occasion de celle-ci, puisqu'il cite des rapports d'inspection rédigés antérieurement : « si cette recommandation inscrite dans nombre de mes rapports [...]».

Ces conceptions initiales s'expriment cependant selon des modalités énonciatives variables, susceptibles de mettre davantage en avant - dans le discours de l'auteur - leur caractère révolu ou toujours d'actualité, attestant dans ce dernier cas d'une continuité affirmée de l'expérience, où connaissances et conceptions empiriques se voient prolongées voire renforcées par les savoirs théoriques, qui ne les invalident pas.

\title{
2.1.2 Des modalités énonciatives variables
}

De ce point de vue, on note que trois documents font part de conceptions initiales présentées comme toujours d'actualité, tandis que le quatrième les présente plutôt comme révolues ; nous verrons que ces variations dans la présentation n'invitent pas nécessairement à une interprétation au pied au de la lettre.

Ainsi, le document 3 présente une conviction affirmée à travers l'emploi d'un passé composé à la voix passive, traduisant le résultat présent d'une action passée : «Depuis que je suis enseignante, j'ai toujours été convaincue [...] ». L'auteur enchérit ensuite : «Cette conviction s'est trouvée renforcée, lors de mes observations, en tant que maitre formateur » [souligné par nous]. L'expérience professionnelle ne se dément pas elle-même, elle ne se verra pas davantage démentie par la recherche conduite au sein du mémoire.

Il en va de même pour le document 4 : «Je pense que l'école maternelle peut jouer un rôle primordial [...] », et pour le document 2, qui inscrit sa conviction dans la durée : « Je suis convaincu et j'essaie de convaincre les enseignants que $[\ldots] »$.

À l'opposé, l'emploi de l'imparfait dans le document 1 traduit une prise de distance par rapport à la conception exprimée, qui laisse penser qu'il y a un avant et un après la recherche, qui vient renouveler l'expérience professionnelle antérieure. : « Nous pensions que [...]. [...] nous menait vers [...]. [...] débutait $[\ldots] \gg$ Dans ce dernier cas, les imparfaits renvoient à des conceptions qui sont présentées comme ayant cours jusqu'au tout début de l'expérience de recherche : "La recherche scientifique débutait avec la prise de conscience que le réel de l'activité ne surgit pas [...] ». La suite est au présent, jusqu'à la fin du mémoire.

Cette présentation révolue des conceptions ne nous conduit cependant pas à les interpréter comme effectivement révolues; dans le document 1, le questionnement cherchera à montrer tout au long du mémoire dans quelle mesure certaines auto-prescriptions des enseignants sont susceptibles d'affecter l'analyse de leur propre pratique enseignante, questionnement qui suppose que cette analyse soit nécessaire pour l'auteur. La recherche montrera ainsi que la question des conditions favorables à cette activité réflexive ne s'exonère pas de la prise en compte de la dimension auto-prescriptive de l'activité enseignante.

D’une manière générale, lorsque les conceptions anciennes sont présentées comme révolues, cela ne signifie donc pas, de notre point de vue, qu'elles sont pour leur auteur obsolètes ou dépassées, mais plutôt 
qu'elles ne sont plus d'actualité dans leur formalisation antérieure. Dans le document 1 en effet, les termes réflexivité/réflexif/réflexive, très présents dans la première partie du mémoire (douze occurrences de la p.1 à la p.22), n'apparaissent plus du tout ensuite, tandis que la notion de développement professionnel apparait en conclusion :

\begin{abstract}
«Ces résultats nous invitent à mesurer l'impact de ces auto-prescriptions sur le développement professionnel pour comprendre en quoi ces nouvelles exigences en gênant la lecture du réel, privent l'enseignant des informations nécessaires pour analyser les performances de sa pratique. » [Doc.1 p. 60, nous soulignons]
\end{abstract}

L'évolution des conceptions se perçoit et se traduit ainsi autant par des déplacements langagiers et des nouveaux modes de formalisation verbale de la pensée, que par des changements ou inversions de postures, ou d'opinions.

On relève par ailleurs d'autres éléments, lexicaux, attestant de cette rupture formelle entre un avant et un après, toujours pour le document $1:$ les termes évoquant la «découverte » (trois occurrences, p.7) et la «prise de conscience» (une occurrence, p.7 également), étroitement associés à l'appropriation par l'auteur des concepts théoriques convoqués.

\title{
2.2 Des déplacements de conception
}

Les découvertes suscitées par le travail scientifique entrainent donc, dans des proportions variables, des déplacements de conceptions, que l'on peut trouver mentionnés explicitement ou beaucoup plus implicitement tout au long du mémoire, donc dans des lieux variés, et formulés également selon des modalités énonciatives variables, qui peuvent traduire diverses formes de dialogisme, de soi à autrui ou de soi à soi.

\subsubsection{Dans la conclusion}

On les trouve donc tout d'abord, et de manière attendue, dans la conclusion, qui vient synthétiser les apports de la recherche, pour la communauté scientifique mais aussi, dans bien des cas, pour l'auteur du mémoire. L'auteur du document 4 établit ainsi une liste des apports de sa recherche concernant le discours enseignant, qui font écho à d'autres travaux cités dans le mémoire, et qui permettent de mieux comprendre ce qui peut faire difficulté dans une classe de maternelle :

- «La non explicitation des enjeux de savoir

- La centration sur les tâches au détriment des objets de savoir

- La doxa pédagogique qui vise à placer les élèves en activité comme si ces dispositifs étaient autosuffisants pour saisir les enjeux d'apprentissage

- La volonté de "donner du sens" selon les enseignants pour enrôler les élèves par l'habillage ludique des activités, mais qui peut rendre opaques les objets de savoir » [Doc.4 p. 54]

On observe ici une forme de transposition des résultats de la recherche en éléments opérationnels pour la classe, et donc pour la formation des enseignants, qui traduit implicitement un déplacement de conception. En effet, ce qui est évoqué en introduction concernant ce qui fait obstacle, dans le discours pédagogique, aux apprentissages n'est pas de même nature que ce qui est dit en conclusion, puisqu'il était question au départ de "langage de proximité », considéré comme un langage qui «n'entrave pas la compréhension », mais ne favorise pas l'appropriation par les élèves d'un langage plus distancié, moins factuel et plus élaboré.

D’une manière un peu différente, on note dans la conclusion du document 3 la présence de nouveaux 
concepts, utiles pour contribuer à mieux comprendre les problèmes soulevés par la recherche menée au sein du mémoire, qui attestent d'une réflexion propre de l'auteur toujours en acte, et un usage des concepts comme outils d'une réflexion qui ne s'exonère plus des apports scientifiques :

Les gestes de métier de ces enseignants experts se construisent grâce à la formation et l'expérience vécue, mais il existe des enseignants novices qui montrent des gestes d'expert dès leur entrée dans le métier. P. Perrenoud (2012) attribue cette qualité à l'habitus de chaque enseignant qui s'élabore dès l'enfance. [Doc.3 p. 49, nous soulignons]

Dans ce cas, les résultats de la recherche sont allés à l'encontre de l'une des hypothèses de l'auteur, supposant que les enseignants chevronnés sont susceptibles de prendre davantage en charge dans leurs propos les enjeux d'apprentissage des activités que des enseignants novices. La recherche ayant montré que l'une des novices du corpus se montrait au contraire experte en la matière, il a fallu replonger dans la théorie pour tenter de rendre compte de cette découverte inattendue. Ici encore, les résultats infirment partiellement des conceptions antérieures, entrainant une reconfiguration de la notion d'expertise, et perceptibles à travers les hypothèses formulées. Les modalités énonciatives retenues pour le dire, dans ce cas particulier, nous paraissent particulièrement significatives :

\begin{abstract}
«À partir des corpus analysés on peut affirmer que la reformulation des enjeux d'apprentissage est bien une caractéristique de l'expertise enseignante. Elle apparait clairement dans le discours des maitres formateurs, mais contrairement à mon hypothèse elle n'est pas réservée aux enseignants chevronnés ou reconnus qualifiés par l'institution. » [Doc. 3 p. 41, nous soulignons]
\end{abstract}

On note ainsi l'emploi du terme réservé, un peu inattendu (on s'attendait davantage à une formule du type « elles ne sont pas l'apanage de »), et un dédoublement du dire à travers la formule reconnus qualifiés par l'institution: or, notre auteur est précisément un formateur titulaire du CAFIPEMF, donc reconnu et qualifié par l'institution. Nous en déduisons que cette découverte comporte peut-être un caractère dérangeant, qui peut déstabiliser l'assise identitaire professionnelle, puisqu'elle amènera ensuite l'auteur à se demander si le fait même que les discours de l'ensemble des formateurs de son corpus attestent d'une prise en charge solide de ces enjeux n'est pas lié à la modestie de l'échantillon.

Enfin, mentionnons la conclusion du document 2, qui traduit des changements de conceptions particulièrement explicites et affirmés :

\begin{abstract}
A la fin de notre travail, nous nous rendons compte qu'il y avait un peu de naïveté de notre part à penser qu'il suffisait de rendre explicites les enjeux des apprentissages pour que les élèves apprennent. A l'issue de cette recherche nous prenons conscience que la problématique de la coconstruction des inégalités scolaires est bien plus complexe que nous l'envisagions au départ [Doc. 2 p. 54, nous soulignons]
\end{abstract}

On y note la présence d'un concept qui ne relève ni de la citation directe ni de la reformulation, mais de l'évocation (Boch, 2013), et qui est évoqué sans mention des références (coconstruction des inégalités scolaires) devenu outil « spontané » de la pensée propre de l'auteur du mémoire, sans pour autant que ces apports scientifiques fassent rupture radicale avec une pensée antérieure : la problématique est dite plus complexe qu'envisagé au départ, il y a donc bien continuité de la pensée dans la complexification, et non abandon de conceptions.

Un peu plus loin, l'auteur fait ainsi référence au temps long de la recherche, qui suppose une évolution inscrite dans la durée, plutôt qu'avec effet immédiat (ce qui ne signifie pas qu'il n'y en ait pas : mais ceux-ci reflètent peut-être des évolutions moins profondes que les effets à plus long terme). Ce propos clôt le mémoire : 


\begin{abstract}
«Il nous reste quant à nous un long chemin à parcourir pour appréhender cette question dans toute sa complexité. Néanmoins, force est de constater que notre recherche nous a permis de nous engager sur ce chemin et de découvrir des perspectives nouvelles en tant que formateur. » [Doc. 2 p. 55-56]
\end{abstract}

\title{
2.2.2 Dans d'autres lieux
}

Les évolutions de conceptions, plutôt explicites dans la conclusion, apparaissent également dans d'autres lieux du mémoire, et peuvent être saisies notamment à partir des transformations de la question de recherche ou des mouvements de la problématisation au sein du mémoire, et à partir de l'usage effectué de certains concepts.

Les mouvements dans la problématisation sont parfois explicitement formulés, comme dans le document 2, montrant ainsi les déplacements intervenus dans la manière de prendre en charge le questionnement :

\begin{abstract}
«La question initiale posée au début de notre recherche était la suivante : Suffit-il de rendre explicite pour les élèves les enjeux d'apprentissage des activités qui leur sont proposées pour qu'ils les perçoivent tous?

A ce stade de notre réflexion, il apparait important d'orienter notre interrogation vers les conditions qui rendent possible la perception des enjeux de la part des élèves, qu'ils soient ou non explicites. Cette formulation nous parait maintenant mieux convenir: Dans quelle mesure les élèves perçoivent-ils les enjeux des activités scolaires explicites ou non dans le discours pédagogique ?» [Doc. 2 p. 16]
\end{abstract}

Ce propos intervient juste après le développement du cadre théorique, qui travaille notamment deux concepts, celui de pédagogie invisible, et celui de malentendu, qui permettent de mieux comprendre le contexte pédagogique qui fait que les élèves ne perçoivent pas tous de la même manière les enjeux des activités qui leur sont proposées. Le travail de ces concepts entraine un déplacement de la question de recherche, qui passe d'une centration sur l'action (suffit-il...pour que?) à une centration sur la compréhension de phénomène (dans quelle mesure les élèves perçoivent-ils... ?).

Les mouvements dans la problématisation apparaissent également dans le document 3, mais plus implicitement, traduisant et entrainant à la fois des changements profonds qui semblent annoncer ceux que nous avons mentionnés à propos de la conclusion de ce mémoire. L'hypothèse d'une plus grande clarté cognitive du discours des enseignants formateurs par rapport aux novices n'étant pas confirmée par la recherche, l'évolution du questionnement, non linéaire, semble refléter une certaine résistance dans le déplacement de conception, non linéaire également.

On observe ainsi un premier déplacement, résultant d'apports théoriques montrant que la mention des enjeux d'apprentissage est assez peu fréquente dans le discours enseignant ; la question se déplace alors de l'enseignant vers l'enseignant expert :
«Après cette lecture, ma question de recherche prenait forme: Quelle place les reformulations facilitant les apprentissages occupent-elles dans le discours enseignant? Après avoir mobilisé quelques éléments théoriques, cette question de recherche s'est précisée et s'énonce maintenant ainsi : La reformulation des enjeux d'apprentissage est-elle une caractéristique du discours des enseignants experts? [Doc. 3 p. 6]

Mais le propos suivant, qui fait suite à une citation montrant, à partir d'un exemple, le caractère invisible de l'enjeu d'apprentissage dans le discours pédagogique, revient à l'enseignant ordinaire : 
"Cette idée nous oblige à considérer les difficultés et différences d'apprentissage des élèves comme des "constructions conjointes de l'enseignant et de l'élève" (Bautier \& Rayou, 2009, p. 94)» [Doc. 3 p. 8 , nous soulignons]

On note ici l'emploi du terme oblige qui traduit une forme de concession théorique, qui relève typiquement de ce que nous nommons, à la suite de Donahue (2004), négociation dialogique, face ici à une idée plutôt dérangeante, qui met en cause l'enseignant dans les difficultés de certains élèves ; la citation intégrée au propos de l'auteur du mémoire peut être lue comme marque de l'adhésion de celui-ci à ce propos pourtant dérangeant. La question de recherche réaffirme dès lors une différence - espérée entre enseignant ordinaire et expert, où l'emploi du terme confirmer autorise à penser que l'auteur croit ou veut croire - à ce moment-là en cette différence :

«Dans le cadre de ma question de recherche, je chercherai à confirmer que les enseignants experts rendent visibles les liens logiques entre les différents discours par leurs reformulations. » [Doc. 3 p. 11]

On retrouve ces éléments modalisateurs qui traduisent des concessions entrainant des évolutions de conceptions dans d'autres mémoires. Dans le document 2 par exemple, à l'issue de la recherche, la concession est prudente, et devient elle-même objet de questionnement :

\begin{abstract}
« Nous pouvons admettre à l'issue de cette recherche que rendre explicite les enjeux permet de lever des malentendus, de permettre à certains élèves de mieux comprendre ce qu'on attend d'eux à l'école, de mieux appréhender les limites souvent très opaques entre travailler et apprendre. » [Doc. 2 p.50, nous soulignons]
\end{abstract}

Mais :

\begin{abstract}
«Les lignes ne sont pas aussi marquées qu'on pourrait trop vite le penser. [...] Ce genre de commentaires nous amène à poursuivre notre réflexion dans deux directions. [Doc. 2 p. 51, nous soulignons]
\end{abstract}

Ici, la recherche engagée peut se poursuivre, elle a suscité d'autres questionnements, et permis de revisiter des conceptions. Mais elle permet aussi d'en tirer, comme pour le document 4, des éléments de réflexion opérationnels pour le métier :

\footnotetext{
« [Un] autre enseignement que nous pouvons tirer de notre recherche est le suivant : si on reproduit jour après jour, mois après mois des séances du type de celles que nous avons proposées au groupe 1, les élèves risquent de ne jamais progresser. » [Doc. 2 p. 51]
}

Dans le document 4, la formulation finale de la question de recherche à l'issue du cadre théorique montre une prise en compte comparable des concepts évoqués, et notamment du dernier, concernant la double visée (auto et allotélique) des dispositifs d'apprentissage scolaires. Les conceptions initiales centrées sur le discours magistral et son rôle se sont déplacées, et prennent une épaisseur conceptuelle qui oblige à les reformuler; l'activité prise dans un sens ordinaire devient dispositif au sens théorique, et la parole enseignante devient discours pédagogique, ce qui suppose de reconnaitre également sa double double visée instructrice et régulatrice :

\footnotetext{
«À l'issue de ce cadre théorique, j'ai reconfiguré ma question de recherche ainsi : $A$ quelles conditions le discours pédagogique permet-il aux élèves de s'approprier la double visée des dispositifs scolaires? » [Doc. 4 p. 16]
} 
Ces exemples montrent le rôle de l'appropriation des concepts dans les mouvements du questionnement des auteurs de mémoires; d'autres permettent cependant d'aller au delà, et dans chacun de ces documents, on constate que l'appropriation des concepts contribue à la robustesse des analyses, comme dans les exemples suivants :

«Cette notion d'auto-prescription est essentielle pour comprendre l'activité
enseignante. Elle deviendra l'organiseur de notre analyse pour catégoriser les choix de
posture des enseignants qui ont participé à cette recherche. » [Doc. 1 p.14]

Pour les réponses des deux élèves suivants nous nous référons à Basil Bernstein qui distingue dans le discours pédagogique le "discours instructeur" et le "discours régulateur". [...] Ces élèves ne parlent que du discours régulateur, le discours instructeur n'est absolument pas perçu. [Doc. 2 p. 39-40]

« Je dois à ce stade de mon analyse prendre appui sur des travaux théoriques afin d'expliquer comment une enseignante novice peut avoir intégré un geste professionnel expert.

Pour le sociologue P. Perrenoud (2012), l'action pédagogique se base pour une grande partie sur l'habitus et non pas sur les savoirs. L'habitus est un ensemble de manière d'être, d'agir et de penser propre à un individu; il est largement inconscient. Cet auteur considère que l'action pédagogique est toujours influencée par l'habitus, selon quatre mécanismes qu'il décrit ainsi : [...] » [Doc. 3 p. 34]

Dans ce dernier cas, le recours à de nouveaux concepts - nous entendons par là des concepts non convoqués dans le cadre théorique du mémoire - a été nécessaire, nous l'avons dit, pour envisager un renouvèlement de l'analyse des données, dont une première version vient contredire les hypothèses de recherche. On note que ces nouveaux concepts sont évoqués de manière complètement intégrée dans le propos de l'auteur du mémoire, modalité énonciative susceptible d'attester de l'adhésion complète de celui-ci à ces derniers, et de l'issue favorable de la négociation dialogique engagée.

Celle-ci se manifeste très visiblement dans chacun des mémoires du corpus à travers l'incertitude d'emploi du pronom choisi pour désigner l'auteur du mémoire. Dans les documents 1 et 2, le pronom librement choisi est le nous; dans les documents 3 et 4 , ce pronom est le pronom je. Pour autant, on note des occurrences de glissement vers le pronom non choisi dans chacun de ces documents - qui sont pourtant des documents remis pour évaluation, et donc en principe soigneusement relus - phénomène que nous avons rencontré presque systématiquement depuis que nous étudions des mémoires, pour les étudiants lauréats d'une licence aussi bien que pour les enseignants en reprise d'étude.

Dans le cas présent, ces glissements s'observent lorsqu'il est question de la pluralité identitaire de l'auteur, simultanément apprenti chercheur (production de savoir), étudiant (restitution de savoir) ou enseignant/formateur (transmission de savoir). Ainsi, le document 1, qui montre trois occurrences de ce glissement, emploie je d'abord dans les remerciements en page 2 (il s'agit ici de l'auteur en tant que personne de chair, pourvue d'un environnement humain, qu'il convoque), puis dans la partie méthodologie, où l'auteur explique comment il a recueilli le corpus, dans la circonscription où il exerce en tant que conseiller pédagogique, enfin dans le paragraphe dont le titre mentionne cette double fonction de conseiller pédagogique et apprenti chercheur, qui clôt la partie méthodologie (où l'auteur explique précisément la nécessité de conserver une vigilance sur la distinction entre posture du formateur et du chercheur). Ces glissements renvoient tous au feuilletage identitaire de l'auteur, attestant ainsi d'une forme de négociation dialogique de soi à soi.

Il en va de même dans le document 2, qui montre deux occurrences de ce glissement, l'une également dans les remerciements, l'autre lorsque l'auteur évoque sa fonction d'IEN. 
Le document 3, qui emploie habituellement le pronom je, laisse paraitre une seule occurrence d'emploi du pronom nous, au début du cadre théorique :

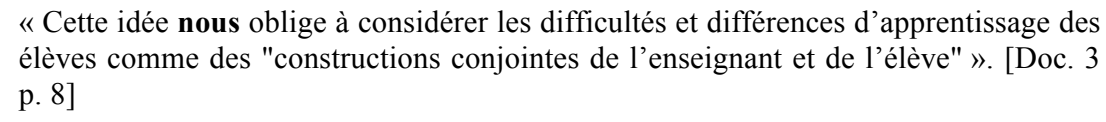

Nous avons analysé ce propos comme susceptible de traduire une forme de concession théorique ; nous ajoutons ici que l'emploi du pronom nous, qui plus est en position d'objet et non de sujet, permet d'atténuer l'implication personnelle de ce même sujet, dont nous avons considéré qu'il pouvait éprouver une certaine réticence vis-à-vis de cette conception dérangeante, en ajoutant une distance rhétorique, et donc en conférant une actualisation moins prégnante ou moins immédiate à cette concession. La fonction de ce glissement relèverait donc ici de la protection du sujet auteur, confronté à des renouvèlements de conception parfois déstabilisants.

Enfin, le document 4, qui emploie également habituellement le pronom je, laisse paraitre quant à lui huit occurrences d'emploi du pronom nous, dont six dans la partie Résultats du mémoire, qui décrit les apports de l'auteur à la communauté scientifique; nous interprétons donc ces occurrences de glissement comme pouvant traduire le sentiment d'intégration dans cette communauté à l'issue de la recherche :

« Nous avons vu dans ce corpus [...]. Nous avons constaté [...]. Nous avons vu [...].

Cependant, nous avons constaté également [...] etc. [Doc. 4 p. 51-53]

\subsection{Pour conclure}

Crahay et al. (2010) évoquent que « bon nombre d'enseignants méconnaissent les résultats des recherches en éducation », et que « leurs croyances s'élaborent donc le plus souvent indépendamment de ce savoir » (ibid., 114). Nos conclusions vont dans le même sens, mais pour cette raison, nous conduisent à réaffirmer avec force que la formation à la recherche et par la recherche des enseignants ou futurs enseignants ne saurait se réduire à une acculturation aux résultats de la recherche : il s'agit bien de les impliquer et de les accompagner dans une démarche réelle de recherche, portée par un questionnement investi, et donnant lieu à des analyses robustes des situations professionnelles. C'est donc tout autant l'apprentissage de méthodologies de recherche, et l'élaboration de catégories d'analyse solides théoriquement ancrées qui permet de reconfigurer le rapport au métier, et non la confrontation incertaine à des résultats de recherche menés ailleurs, par d'autres, et sans que l'on puisse savoir comment ces résultats ont été produits.

Ce travail réel d'élaboration scientifique, qui engage l'auteur du mémoire simultanément en tant que chercheur et en tant qu'enseignant dans une négociation complexe avec lui-même, d'autant plus puissante qu'elle se nourrit des savoirs théoriques antérieurement produits sur la question, permet, à certaines conditions, une évolution des conceptions antérieures. Quelles sont ces conditions ? Et en quoi consiste l'évolution de ces conceptions?

Concernant le premier point, il nous parait intéressant de reprendre le propos de Crahay et al. qui rappellent, à la suite d'Ashton (1984), que « les enseignants caractérisés par une haute perception de leurs compétences (high self-efficacy) sont davantage enclins à changer leur pratique que les autres » $(O p$. cit., $2010,95)$. Nous pensons en effet que ce principe est susceptible de s'appliquer aussi aux changements de conceptions, et que les enseignants formateurs dont nous avons étudié les travaux, dont les compétences sont reconnues et attestées par l'obtention du CAFIPEMF, qui les rend officiellement aptes à la fonction de formateurs, sont de ce fait plus enclins à revisiter ces conceptions, ce dont atteste en partie leur inscription volontaire dans ce master. Les déplacements constatés sont donc rendus possibles notamment 
par l'expérience d'une pratique vécue comme opérationnelle, et non par les seuls obstacles qu'elle n'a pas manqué de faire apparaitre.

D'autre part, ces pratiques opérationnelles sont sous-tendues par des conceptions dont nous avons montré qu'elles sont reconfigurées, mais non remises en cause radicalement par l'élaboration scripturale scientifique, point qui nous apparait comme un facteur déterminant pour penser la question de l'évolution des conceptions des enseignants.

Précisons à ce propos que si toute mention d'un point de vue ne peut être considérée en soi comme l'expression d'une conception, ces mentions, qui font figures de parties émergées de l'iceberg, sont cependant susceptibles de donner accès à des conceptions ; au plan méthodologique, il nous semble donc utile de mentionner que les évolutions de conceptions nous semblent pouvoir être saisies à travers des objets très divers, à condition de disposer d'un faisceau d'éléments, ce que favorise l'écriture longue.

L'étude de ces mémoires montre ensuite que les déplacements de conception s'inscrivent dans une continuité de l'expérience: les questions de recherche viennent reconfigurer des questionnements professionnels sous tendus par des conceptions fortes, questionnements donc déjà présents pour les quatre auteurs, qui les ont cependant abordés autrement dans le mémoire, et avec d'autres méthodes et démarches d'analyse, ce qui a conduit à les formaliser autrement. Cette nouvelle formalisation ne se produit que conjointement à l'écriture scientifique, et c'est là une dimension qui nous semble devoir être largement soulignée. Car si nous considérons qu'au delà de l'acculturation à des résultats, la formation des enseignants par la recherche ne peut être efficace qu'à condition que ceux-ci s'approprient des méthodologies de recherche, la question cruciale du rôle de l'écriture dans ce processus, essentielle, ne nous semble que rarement mise en avant.

En ce qui concerne la nature des conceptions revisitées, elles sont de deux ordres. Elles concernent d'une part le métier au sens large, et peuvent porter sur l'apprentissage, la pratique, les dispositifs et le discours pédagogiques, la discipline, le contexte d'exercice etc. En l'occurrence, trois des mémoires étudiés portent sur le discours pédagogique, et un sur les organisateurs de la pratique pédagogique; tous concernent donc le pôle « enseignant», pôle particulièrement privilégié dans l'ensemble des mémoires de formateurs que nous avons étudiés.

D'autre part, l'évolution des conceptions porte aussi - et surtout, pensons-nous - sur ce qui peut contribuer à l'analyse des situations pédagogiques, et ce point nous semble lui aussi déterminant. Les enseignants qui ont en effet mené à terme leur projet de recherche, et se sont emparés des méthodologies scientifiques d'analyse, peuvent difficilement recourir ensuite à des méthodes d'analyse fondées sur la seule expérience professionnelle. Il y aurait lieu de chercher à évaluer dans quelle proportion cette affirmation est pertinente, mais nous pouvons déjà indiquer que les déplacements de conceptions observés au sein de ces quatre mémoires sont présentés, plus ou moins explicitement, comme le début d'un processus intellectuel entrainant un renouvèlement de la réflexion engagée antérieurement, et non comme l'achèvement de ce processus. En termes de perspectives scientifiques, il parait donc porteur d'envisager des investigations concernant l'évolution des conceptions après le mémoire.

\section{Références bibliographiques}

Ashton, P. (1984). Teacher efficacy: A motivational paradigm for effective teacher education. Journal of teacher education, voL. $35, n^{\circ} 5,28-32$.

Boch, F. (2013). Former les doctorants à l'écriture de la thèse en exploitant les études descriptives de l'écrit scientifique. Linguagem em (Dis)curso, Tubarao, SC, 13-3, 543-568.

Bres, J. (2005). Savoir de quoi on parle : dialogue, dialogal, dialogique ; dialogisme, polyphonie... In Bres, J., Haillet, P.-P., Mellet, S., Nolke, H., Rosier L. (dir.). Actes du colloque de Cerisy: Dialogisme et polyphonie. Approches linguistiques. Bruxelles : De Boeck-Duculot, 47-61. 
Crahay, M., Wanlin, P., Issaieva, I., et Laduron I. (2010). Fonction, structuration et évolution des croyances (et connaissances) des enseignants. Revue française de pédagogie, 85-129.

Crinon, J. (dir.), (2003). Le Mémoire professionnel des enseignants. Observatoire des pratiques et levier pour la formation. Paris : L'Harmattan.

Crinon, J. \& Guigue, M. (2006). Écriture et professionnalisation, Revue française de pédagogie, n 156, 117-169.

Crinon, J. \& Guigue, M. (2004). Écrire ses pratiques dans des mémoires professionnels. In Blanchard-Laville, C. \& Fablet, D. (dir.). Écrire les pratiques professionnelles. Paris : L'Harmattan, 167-198.

Delarue-Breton C. (2014a). Créativité, au(c)torisation et dialogisme : le mémoire de master MEEF, miroir de l'expérience psychique ? Actes du IVème Congrès Mondial de Linguistique Française (CMLF), Symposium « Linguistique de l'écrit, linguistique du texte, sémiotique, stylistique », Berlin 19-23 juillet.

Delarue-Breton, C. (2014b). Le mémoire de master MEEF: un nouveau genre universitaire, porteur de (trans)formation de l'identité professionnelle ? Diversité, 177, 50-55.

Delarue-Breton, C. et Crinon, J. (2015). De l'usage des concepts dans les mémoires de master Métiers de l'enseignement. Le Français Aujourd'hui, 188, 79-88.

Delarue-Breton, C. (2016). Créer le déjà-là, un paradoxe fécond pour penser l'activité scripturale et scientifique de l'auteur de mémoire de master MEEF. Pratiques, 169-170.

Delarue-Breton, C. \& Dolignier, C. (2016). Posture "seconde" et évolution des croyances des enseignants en formation initiale : le rôle de la recherche. Actes du colloque Le Printemps de la recherche en ÉSPÉ, Paris, 23 mars.

Donahue, C. (2001). Effets de l'écrit sur la construction du sujet textuel à l'université. Spirale, 28, 75-108.

Donahue, C. (2004). Évolution d'une métaphore-modèle, analytique et pédagogique : les communautés discursives et la composition theory aux Etats-Unis. Les cahiers Théodile $n^{\circ} 5$, 93-117.

Frier, C. (1998). Profils de lecteurs et modalités d'approche des textes de spécialité à l'Université, Lidil, 17, 65-79.

Rabatel, A. (2013). Positions, positionnements et postures de l'énonciateurs. Linha d'Agua, 26-2, 159-183.

Winnicott, D.W. (1975/1971). Jeu et réalité. L'espace potentiel. Traduction C. Monod et J.-B. Pontalis, 1975. Paris : Gallimard.

\footnotetext{
${ }^{1}$ Master MEEF : master mention Métiers de l'Enseignement, de l'Éducation et de la Formation. Les étudiants dont nous parlons sont lauréats d'une licence, et se destinent au métier de professeur des écoles (l'école primaire accueille en France les enfants de 3 à 11 ans). Le master MEEF mention 1 vise à les préparer simultanément au diplôme de master, au concours de recrutement des professeurs des écoles et au métier d'enseignant.

${ }^{2}$ Le cursus universitaire européen dit LMD est structuré en trois temps : niveau Licence (trois ans), niveau Master (deux ans) et niveau Doctorat (trois à six ans).

${ }^{3} \mathrm{JORF}^{\circ} 0200$ du 29 août 2013, texte 48, p. 4627.

${ }^{4}$ IUFM : Institut Universitaire de Formation des Maitres

${ }^{5}$ Les étudiants inscrits en master MEEF dans l'académie de Créteil sont tenus de rédiger un mémoire de master en deux ans; à la fin du Master 1, ils doivent rendre un écrit de 50.000 signes constituant un projet de recherche (définition d'un cadre théorique et méthodologie envisagée), intitulé note de recherche, qui sera repris et transformé en M2 pour devenir un mémoire qui sera soutenu à la fin du Master 2. Le mémoire final est un mémoire de format relativement court (100.000 signes), qui décrit la recherche qui s'élabore conjointement à lui.
} 